\title{
Trimer superfluid and supersolid on two-dimensional optical lattices
}

\author{
Wanzhou Zhang, , * Yuan Yang, ${ }^{2}$ Lijuan Guo, ${ }^{1}$ Chengxiang Ding, ${ }^{3}$ and Tony C Scott ${ }^{1}$ \\ ${ }^{1}$ College of Physics and Optoelectronics, Taiyuan University of Technology Shanxi 030024, China \\ ${ }^{2}$ College of Materials Science and Engineering, Taiyuan University of Technology, Taiyuan 030024 \\ ${ }^{3}$ Department of Applied Physics, Anhui University of Technology, Maanshan 243002, China
}

(Dated: June 19, 2018)

\begin{abstract}
By the photoassociation method, the trimer superfluid phase disappears in the one dimensional state-dependent optical lattice if the ratio of the three-body interaction $W$ to the trimer tunneling $J$ is kept at $W / J=12$ [Phys Rev A. 90, 033622(2014)]. To search for a trimer superfluid and trimer supersolid, we load the cold atom into two-dimensional lattices, whose coordinate number $z$ and kinetic energy $-z J$ are respectively larger and lower than those of a one dimensional lattice. Herein, we study the Bose-Hubbard model, which has an additional trimer tunneling term, a threebody interaction and a next-nearest repulsion. The on-site trimer and trimer superfluid exist if the on-site two-body repulsion and three-body repulsion are smaller than some thresholds. With atom-tunneling terms, the phase transitions from trimer superfluid phase to both atom superfluid and atom supersolid phases are first order. With $W / J=12$, in a one dimensional lattice, the trimer superfluid phase does not exist at all. In contrast, the trimer superfluid phase, exists in the lower density regions $0<\rho<2$ on the square lattice if $J$ is not very large. The trimer superfluid phase emerges in a wider range $0<\rho<3$ in the triangular lattice, or in the cubic lattice $(z=6)$. When the three-body interaction is turned on, a trimer supersolid phase emerges due to the classical degeneracy between the quasi-trimer solid and the trimer solid being broken by the quantum fluctuation. The phase transitions from the trimer supersolid phase to the quasi trimer solid are first order and the phase transition from the trimer supersolid phase to trimer solid is continuous. Our results, obtained by mean-field and quantum Monte Carlo methods, will be helpful in realizing the trimer superfluid and supersolid by cold atom experiments.
\end{abstract}

PACS numbers: $67.85 . \mathrm{Hj}, 03.75 .-\mathrm{b}, 67.80 . \mathrm{kb}$

\section{INTRODUCTION}

So far, trimer states have been realized in cold atom experiments, by several alkali metal atoms, such as ${ }^{7} \mathrm{Li}$ [1, 2], ${ }^{39} \mathrm{~K}$ [3], ${ }^{85} \mathrm{Rb}$ [4], ${ }^{133} \mathrm{Cs}$ [5] and Li-Cs [6]. The schemes involving magnetic Feshbach resonance (FR) [1] and photoassociation (PA) [7, 8], or combining the latter two methods [9] allow us to control the binding energies of the trimers in a wide range from $0 \mathrm{~Hz}$ to several $\mathrm{MHz}$. Although the trimers in current experiments [1 $[6]$ are just trapped in a local well within particular few-body systems, studies can provide us some universal properties $[6,10,11]$ and a possibility to study the correlation effects for the trimers in the optical lattice.

The strongly-correlated effects lead to the trimer superfluid(TSF) [12 16], in which the laser-driven threeatom combinations move together to the nearby lattice, without any separation. In some parameter regions, the three-atom combination on a particular site has a nonzero correlation with the holes at an infinite distance. Consequently, the off-diagonal long-range order (ODLRO) [17] takes form, i.e., $\lim _{r \rightarrow \infty} a_{i}^{\dagger 3} a_{i+r}^{3} \neq 0$.

The Bose-Hubbard model, with trimer tunneling terms and other density-dependent tunneling terms, has been proposed by two of the authors [15]. However, the

\footnotetext{
*Corresponding author: zhangwanzhou@tyut.edu.cn
}

nearest-neighbor repulsion, which can be realized by dipolar interaction [18], leads to various interesting solid phases, with broken translation-symmetry [19]. Furthermore, if the solid order coexists with the ODLRO simultaneously, then the supersolid (SS) phase emerges. Previously, various novel SS phases were observed, such as the pair SS (PSS) phase on the bilayer lattice 20], the triangular lattice with pair hopping terms 21 23], in two-species systems 24], f-wave SS phases 25] and molecular systems [26]. However, it is still unknown whether or not, the trimer supersolid (TSS), a type of supersolid with TSF order exists in real systems.

On the other hand, there is a condition for the existence of the TSF phase in the state-dependent lattice 15] as the three-body interaction $W$ will compete with the kinetic energy $-z J$. The larger the coordination number is, the lower the kinetic energy becomes. In the one dimensional system, without the magnetic Feshbach resonance, the ratio will be kept at $W / J=12$, at which the TSF phase will disappear and the Mott insulator will appear. Although modulating $W$ independently can be achieved by combining the FR and PA methods, this will add to the complexity of the experiment.

Fortunately, for the two dimensional system, the TSF phase still exists at the point $W / J=12$ [15] when using only the PA method. Therefore, it is easier to find the TSF phase in the two dimensional system than in the one dimensional system in cold atom experiments and consequently we will choose a square lattice at $z=4$ and a triangular lattice at $z=6$ as the platforms by which to 
study the TSF and the TSS phases.

The outline of this work is as follows. Section [I] shows the Hamiltonian model and the classical limit of the model on both a square and triangular lattice. Section III shows the mean field (MF) and quantum Monte carlo (QMC) methods and useful observables. We then present the ground-state phase diagram and the TSF phase in the square lattice in Sec. IV] and results for the triangular lattice in Sec. V] for various cases. Concluding comments are made in Section VI

\section{MODEL, CLASSICAL LIMIT}

\section{A. Model}

The starting point of our study is the Hamiltonian given by:

$$
\begin{aligned}
H= & -t \sum_{\langle i, j\rangle}\left(a_{i}^{\dagger} a_{j}+a_{j}^{\dagger} a_{i}\right)-J \sum_{\langle i, j\rangle}\left(a_{i}^{3 \dagger} a_{j}^{3}+a_{j}^{3 \dagger} a_{i}^{3}\right) \\
& +\frac{U}{2} \sum_{i} n_{i}\left(n_{i}-1\right)+V \sum_{\langle i, j\rangle} n_{i} n_{j}-\mu \sum_{i} n_{i} \\
& +\sum_{i} \frac{W n_{i}\left(n_{i}-1\right)\left(n_{i}-2\right)}{6}
\end{aligned}
$$

where $\langle i, j\rangle$ denotes the nearest-neighbor sites, $a_{i}^{\dagger}\left(a_{i}\right)$ is the boson creation (annihilation) operator at site $i$, and $n_{i}=a_{i}^{\dagger} a_{i}$ the boson number operator. The term $t(J)$ is the single-atom (trimer) hopping amplitude, $U$ the onsite interaction, $\mu$ the chemical potential, $V$ the nearestneighbor repulsion, and $W$ the three-body interaction. These parameters in the Hamiltonian can be expressed as:

$$
\begin{aligned}
J & =2 \frac{\Omega_{1} \Omega_{2}}{\Delta} \\
U & =U_{0}-\frac{12}{\Delta}\left(\Omega_{1}^{2}+\Omega_{2}^{2}\right) \\
W & =W_{0}+\frac{12}{\Delta}\left(\Omega_{1}^{2}+\Omega_{2}^{2}\right) \\
V & =U_{0} e^{-\frac{3}{2} \pi^{2}} \sqrt{\frac{V_{0}}{E_{R}}}
\end{aligned}
$$

where $U_{0}$ and $W_{0}$ are the traditional two-body repulsion term [27] and three-body repulsion term respectively, and $\Delta$ is the detuning parameter, $V_{0} / E_{R}$ is the ratio between the depth of the optical well to the recoil energy of the atoms. The ratio $W / J$ becomes a fixed value 12 for any values of $\Delta$ due to $W_{0}$ being very weak and therefore negligible. The coupling between atoms is defined as:

$$
\Omega_{1(2)}=\Omega \int d \mathbf{r} w_{m}^{*}\left(\mathbf{r}-\mathbf{r}_{\mathbf{i}_{\mathbf{m}}}\right) w_{a}\left(\mathbf{r}-\mathbf{r}_{\mathbf{i}_{\mathbf{a}}\left(\mathbf{i}_{\mathbf{a}}+\mathbf{1}\right)}\right)^{3},
$$

where $w_{a(m)}\left(x-x_{i}\right)$ are the ground state Wannier functions for an atom (a trimer) in an optical lattice potential. If $t$ or $J$ dominates the Hamiltonian, the system sits in the atom superfluid (ASF) or TSF phase. If the remaining interactions dominate the Hamiltonian, the system will enter into the solid phases or Mott insulator (MI) phases. In some regions, we also expect a TSS phase.

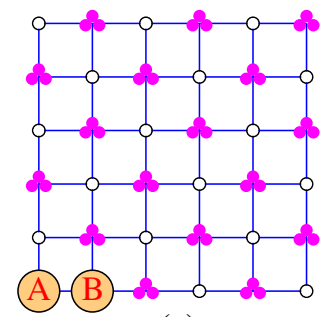

(a)

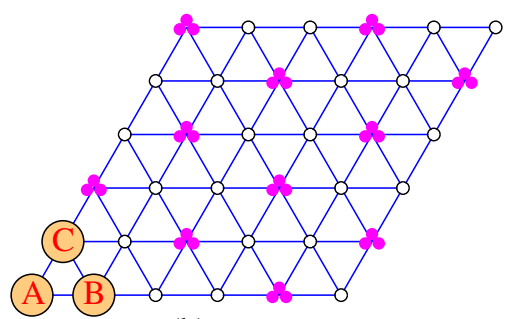

(b)
FIG. 1: (Color online) (a) Trimer solid phase $(0,3)$ on the square lattice, where the numbers $\left(n_{A}, n_{B}\right)$ denote the boson occupation numbers in the sublattices. The three magenta symbols and empty symbols denote trimers and empty sites, respectively. (b) Trimer solid phase $(0,0,3)$ on the triangular lattice. The trimer solid phase $(0,3,3)$ and other quasi-trimer solids such as $(0,1,3)$ and $(2,3,3)$ are not shown.

\section{B. Classical limit: trimer solid}

This section shows that the trimers still exist if the two-body and three-body interactions are in reasonable ranges.

In the classical limit, $t=0, J=0$ and the nearest neighborhood repulsion $V$ in the Hamiltonian of Eq. (1) breaks translational symmetries of the original lattice. A minimum cell in the solid phases is composed of two (three) sublattices $A$ and $B(A, B$ and $C$ ) for a square (triangular) lattice as shown in Fig. 1, We use $n_{A}$, $n_{B}$, and $n_{C}$ to denote the occupation numbers, and the Hamiltonian in a cell becomes:

$$
H=\sum_{i} H_{i}+H_{V}
$$

where the on-site Hamiltonian is:

$$
H_{i}=\frac{U n_{i}\left(n_{i}-1\right)}{2}+\frac{W n_{i}\left(n_{i}-1\right)\left(n_{i}-2\right)}{6}-\mu n_{i},
$$

and the sum is taken over the sites in the cell. Here, the interaction term is: $H_{V}=\frac{z V}{2}\left(n_{A} n_{B}+n_{B} n_{A}\right)$ for the square lattice and:

$$
H_{V}=\frac{z V}{2}\left(n_{A} n_{B}+n_{B} n_{C}+n_{C} n_{A}\right)
$$

for the triangular lattice. The coordination number $z$ is 4 for the square lattice and 6 for triangular lattice.

We can obtain the phase boundaries between various ordered phases by comparing the energies per unit cell in the phases. The phase diagrams of the square lattice and the triangular lattice are shown in the upper and lower row respectively. We show the phase diagram in 
the plane $(U / V, \mu / V)$ with $W=0$ in Figs. 2 (a) and (c), and the phase diagram $(W / V, \mu / V)$ with $U=0$ in Figs. 2 (b) and (d).
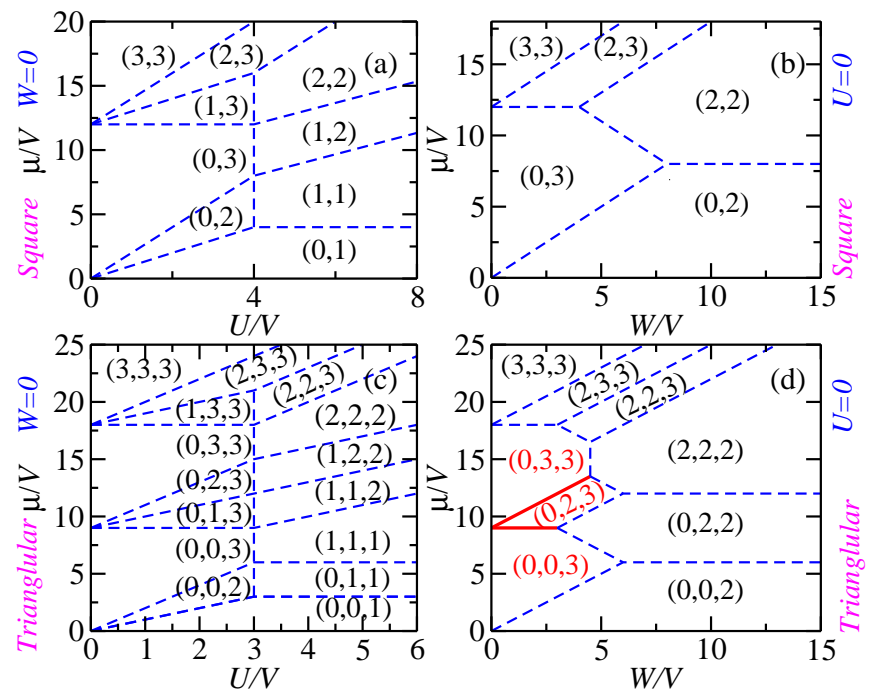

FIG. 2: (Color online) Classical limit $(t=0, J=0)$ of the $\mathrm{BH}$ model with (left) and without (right) the three-body interaction. The upper row is for the square lattice and the lower row is for the triangular lattice. (d) The trimer solids $(0,3,3)$ and $(0,0,3)$ are separated with $W / V \neq 0$ while the two solids are degenerate with the quasi solid $(0,2,3)$ along the solid lines.

In the square lattice, as shown in Fig. 2(a), only one solid phase $(0,3)$ exists with $U=0$. Due to the breaking of the trimer by the nonzero two-body interaction, quasi trimer solid phases $(1,3)$ and $(2,3)$ emerge as $U / V<4$. The remaining on-site trimers are broken and solid phases $(0,1)$ and $(1,2)$ exist without trimers if $U / V \geq 4$. In the limit $U \rightarrow \infty$, the system reduces to hardcore bosons, and only two possible occupation numbers 0 and 1 are allowed, which are not shown. Similarly, in Fig. 2(b), the maximum range of the trimer solid $(0,3)$ is $W / V<7.5$.

Figs. 2(c) and (d) show the effects of $U$ and $W$ on the trimers in the triangular lattice. The trimer solids $(0,3$, $3),(0,0,3)$ and the quasi trimer solid $(0,2,3)$, which are of special interest, exist in the range $U / V<3$ and $W / V<5$ as shown in Figs. 2(c) and (d). With $U=0$ and $W=0$, the system resembles the hardcore $\mathrm{BH}$ model on the triangular lattice, just by replacing the solids $(0,0,3)$ and $(0,3,3)$ with $(0,0,1)$ and $(0,1,1)$ respectively. Due to the geometrical frustration, in the classical limit, the solids $(0,0,1)$ and $(0,1,1)$ are degenerate at the holeparticle symmetric point and the system has a perfect solid order with the wave vector $\mathbf{Q}=(4 \pi / 3,0) 228,29]$. Turning on the hopping terms, the solid orders would be partially broken by the quantum fluctuation with the SS order forms coexisting with the solid orders.

As shown in Fig. 2(d), the two solids $(0,0,3)$ and $(0,3,3)$ are not degenerate and are separated by the solid $(0,2,3)$ in the phase diagram with $W / V \neq 0$. One may infer that the supersolid does not exist as the degener- acy is broken. However, the quasi trimer solid $(0,2,3)$ is degenerate with the solids $(0,0,3)$ and $(0,3,3)$ along the solid lines in Fig. 2(d). With trimer hopping, it is necessary to check the possibility for the existence of the TSS phase, which will be discussed in Sec. VB.

\section{METHODS AND THE MEASURED QUANTITIES}

\section{A. Mean-field method}

By the decoupling approximation [30 32] defined by:

$$
O_{i}^{\dagger} O_{j}=\left\langle O_{i}^{\dagger}\right\rangle O_{j}+O_{i}^{\dagger}\left\langle O_{j}\right\rangle-\left\langle O_{i}^{\dagger}\right\rangle\left\langle O_{j}\right\rangle
$$

we can transform the model Eq. (1) into the mean-field form by decoupling the terms $a_{i}^{\dagger} a_{j}, a_{i}^{3 \dagger} a_{j}^{3}$ and $n_{i} n_{j}$ into single site forms. The Hamiltonian on the triangular lattice can be considered as the sum over local Hamiltonian operators on the three sublattices $A, B$, and $C$. The Hamiltonian on the sublattice $A, h_{A}$, is as follows:

$$
\begin{aligned}
h_{A}= & -3 t\left(a_{A}^{\dagger}+a_{A}\right)\left(\Psi_{B}^{a}+\Psi_{C}^{a}\right)+3 t \Psi_{A}^{a}\left(\Psi_{B}^{a}+\Psi_{C}^{a}\right) \\
& -3 J\left(a_{A}^{3 \dagger}+a_{A}^{3}\right)\left(\Psi_{B}^{t}+\Psi_{C}^{t}\right)+3 J \Psi_{A}^{t}\left(\Psi_{B}^{t}+\Psi_{C}^{t}\right) \\
& +3 V n_{A}\left(\rho_{B}+\rho_{C}\right)-3 V \rho_{A}\left(\rho_{B}+\rho_{C}\right) / 2 \\
& +U\left[n_{A}\left(n_{A}-1\right)\right] / 2-\mu n_{A} \\
& +W n_{A}\left(n_{A}-1\right)\left(n_{A}-2\right) / 6
\end{aligned}
$$

where $\Psi^{a}=\langle a\rangle, \Psi^{t}=\left\langle a^{3}\right\rangle$, and $\rho=\langle n\rangle$. For the square lattice, we only need to replace the above Hamiltonian with the following:

$$
\begin{aligned}
\hat{h}_{A}= & -4 J \Psi_{B}^{t}\left(\hat{a}_{A}^{3}+\hat{a}_{A}^{\dagger 3}\right)+\hat{n}_{A}\left(-\mu+4 \rho_{B} V\right) \\
& +4 J \Psi_{A}^{t} \Psi_{B}^{t}-2 \rho_{A} \rho_{B} V \\
& +U\left[n_{A}\left(n_{A}-1\right)\right] / 2-\mu n_{A} \\
& +W n_{A}\left(n_{A}-1\right)\left(n_{A}-2\right) / 6 .
\end{aligned}
$$

One can obtain the Hamiltonian operators $h_{B}$ and $h_{C}$ by cyclic permutation of the subscripts $A, B$, and $C$.

We resolve the Hamiltonian operators and obtain the solutions of order parameters iteratively. The expectation values of the creation (annihilation) operators are considered as variational parameters in a manner similar to that of references 33 35].

One can initiate a multiple-group of trial parameters $\left(\Psi_{j}^{a}, \Psi_{j}^{t}, \rho_{j}\right)$, where $j=A, B$ and $C$, and then solve the eigenvalue equation of $h_{A}$. We input the newly calculated quantities $\Psi_{A}^{a}, \Psi_{A}^{t}$, and $\rho_{A}$ into $h_{B}$ and $h_{C}$ sequentially. We iterate until the order parameters converge. In order to filter out the metastable states, we save the states of the lowest ground-state energy, by which we get the ground-state phase diagrams.

In Tab. I we show the values of the order parameters for several typical phases. The solid orders denoted by $\Delta \rho, \Delta \Psi^{a}$ and $\Delta \Psi^{t}$ are defined by:

$$
\Delta O=\sum_{i=A, B, C}\left|O_{i}-\bar{O}\right|, \quad \bar{O}=\frac{1}{3} \sum_{i=A, B, C} \bar{O}_{i},
$$


TABLE I: Values of the order parameters for typical phases.

\begin{tabular}{c|cccccc}
\hline \hline & ASF & Solid & ASS & TSF & TSS & MI \\
\hline$\Psi^{a}$ & $\neq 0$ & 0 & $\neq 0$ & 0 & 0 & 0 \\
$\Psi^{t}$ & $\neq 0$ & 0 & $\neq 0$ & $\neq 0$ & $\neq 0$ & 0 \\
$\Delta \rho$ & 0 & $\neq 0$ & $\neq 0$ & 0 & $\neq 0$ & 0 \\
$\Delta \Psi^{a}$ & 0 & $\neq 0$ & $\neq 0$ & 0 & 0 & 0 \\
$\Delta \Psi_{t}^{t}$ & 0 & $\neq 0$ & $\neq 0$ & 0 & $\neq 0$ & 0 \\
\hline$\rho_{s}^{a}$ & $\neq 0$ & 0 & $\neq 0$ & 0 & 0 & 0 \\
$\rho_{s}^{t}$ & $\neq 0$ & 0 & $\neq 0$ & $\neq 0$ & $\neq 0$ & 0 \\
$S(\mathbf{Q}) / N$ & 0 & $\neq 0$ & $\neq 0$ & 0 & $\neq 0$ & 0 \\
\hline \hline
\end{tabular}

where $O$ can be replaced by $\rho, \Psi^{a}$, and $\Psi^{t}$. More details are available in the work of references [15, 23, 30].

\section{B. Improved Quantum Monte Carlo method}

To check the results obtained from the MF method, we simulate the model (1) using the stochastic series expansion (SSE) QMC method [36] with the directed loop update 37. To simulate the pair superfluid phase, the algorithm is improved by allowing the head of a directed loop to carry a pair [38 41] of creation (annihilation) operators $a^{2 \dagger}\left(a^{2}\right)$. In the present work, to make the atom trimers active, we let the loop heads carry trimer creation (annihilation) operators $a^{3 \dagger}\left(a^{3}\right)$.

To distinguish the ASF and the TSF states, we define two types of superfluid stiffness $\rho_{s}^{\alpha}$ as the order parameter [15, 42], which can be determined as:

$$
\rho_{s}^{\alpha}=\frac{1}{2}\left(\rho_{s}^{\alpha, x}+\rho_{s}^{\alpha, y}\right),
$$

where $\rho_{s}^{\alpha, x}$ and $\rho_{s}^{\alpha, y}$ are the superfluid stiffness along $x$ an $y$ directions respectively. In the above equation, $\rho_{s}^{\alpha, x}$ is defined as

$$
\rho_{s}^{\alpha, x}=\frac{L^{2-d}\left\langle W(\alpha, x)^{2}\right\rangle}{2 d \beta(9 J+t)} .
$$

If $\alpha=t, W(\alpha, x)$ is the winding number which can be divided without remainders. If $\alpha=a, W(\alpha, x)$ is the winding number which cannot be divided without remainders. The definition of $\rho_{s}^{\alpha, y}$ is similar with that of $\rho_{s}^{\alpha, x}$. For a TSF phase, we define the order parameter $\rho_{s}^{t}>0$ and $\rho_{s}^{a}=0$. For an ASF phase, $\rho_{s}^{a}>0$ and $\rho_{s}^{t}=0$. The parameters $d$ and $L$ are respectively the system dimensionality and size, and $\beta$ is the inverse temperature.

The structure factor is defined to characterize the solid order:

$$
S(\mathbf{Q}) / N=\left\langle\rho_{\mathbf{Q}} \rho_{\mathbf{Q}}^{\dagger}\right\rangle,
$$

where $\rho_{\mathbf{Q}}=(1 / N) \sum_{i} n_{i} \exp \left(i \mathbf{Q} \mathbf{r}_{i}\right)$. The trimer solids $(0$, $0,3)$ and $(0,3,3)$ share the same ordering at the wave vector $\mathbf{Q}=(4 \pi / 3,0)$ and the same value 1 in the perfect ordering. In the square lattice, the wave vector should be $\mathbf{Q}=(\pi, \pi)$ for the solid $(0,3)$.
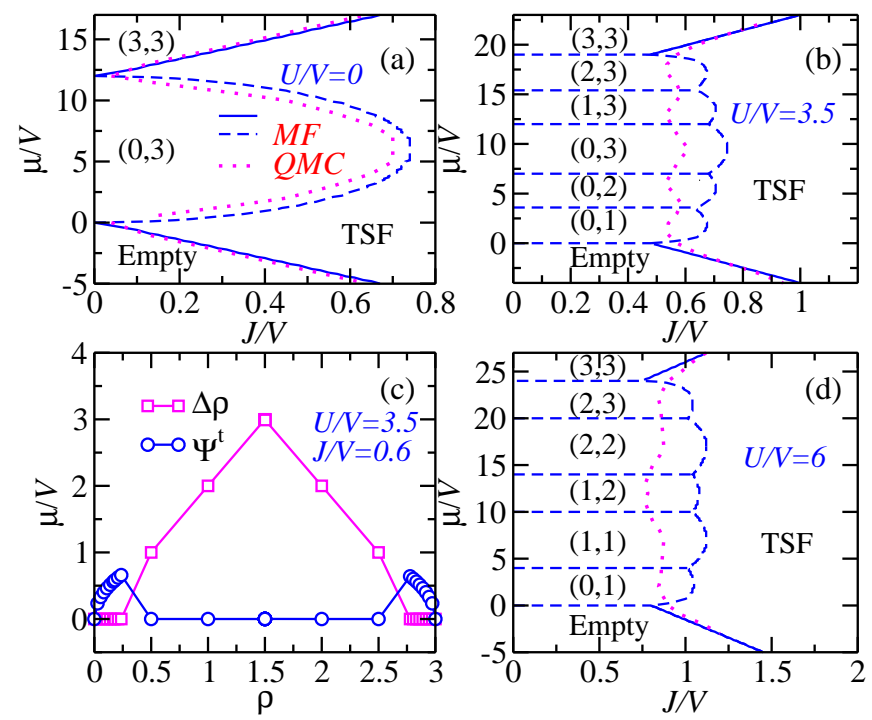

FIG. 3: (Color online) MF and QMC ground-state phase diagrams for $U / V=0$ (a), 3.5 (b), and 6 (d), respectively. Soliddashed lines by MF method denote first-order phase transitions, whereas solid lines represent the continuous phase transitions. The doted lines are obtained by the QMC method. (c) The hole-particle symmetry is shown by plotting $\Delta \rho$ and $\Psi^{t}$ as function of $\rho$.

\section{ASS AND TSF ON THE SQUARE LATTICE}

\section{A. Solid and TSF with $t / V=0$ and $W / V=0$}

The phase diagrams for $U=0,3.5$, and 6 , are shown in Figs. 3 (a), (b) and (d), respectively. Several typical trimer solid phases are found. Also, with larger $J / V$, a TSF phase emerges. The phase transition from the solid phase to the TSF phase is first order and the phase transitions from the empty phase to the TSF (MI) phase are continuous. No supersolid phase is found, which is consistent with the hardcore bosons in biparite lattice 43 , 44]. These results are obtained by solving the equations described in section III A.

To understand the phase diagram, we also try to solve the MF Hamiltonian analytically. In the TSF phase, there is no symmetry broken between the two sublattices $A$ and $B$ and these two sublattices are equivalent, so we can set $\Psi_{A}^{t}=\Psi_{B}^{t}$ and $\rho_{A}=\rho_{B}$ in Eq. (7). In this way, the number of the variables will be reduced. By solutions for the minimum value i.e. the lowest eigenvalue of the Hamiltonian in Eq. (7), we find that the density is:

$$
\rho=\frac{24 J+3 \mu}{16 J+12 V}
$$

and the trimer superfluid order parameter is:

$$
\Psi^{t}=\frac{\sqrt{3(8 J+\mu)(8 J+12 V-\mu)}}{2 \sqrt{2}(4 J+3 V)} .
$$

From Eqs. (12) and (13), we can obtain the phase boundaries between the TSF phase and the solid(MI) phases. 
Letting $\Psi^{t}=0$ in Eq. (13), we get the straight phase boundary $\mu / V=-8 \mathrm{~J} / V$ from the TSF phase to the empty phase, and the straight phase boundary $\mu / V=$ $8 J / V+12$ from the TSF phase to the MI phase. This can also be understood by the single particle theory 15$]$.

The boundary line from the solid $(0,3)$ to the TSF phase, can also be obtained. By substituting Eq. (12) and Eq. (13) into the lowest eigenvalue, we get:

$$
E=-(24 J+3 \mu)^{2} /(16 J+12 V) .
$$

By letting $E=-1.5 \mu$, where the right side of the previous equation is the energy per site in the solid $(0,3)$. The boundary line of the solid phase to the TSF phase is obtained as follows:

$$
\mu / V=6 \pm 2 \sqrt{9-16 J^{2} / V^{2}},(J / V \leq 3 / 4) .
$$

The equation above is consistent with the numerical solutions. The triangular symbols in the phase diagram are the data obtained by QMC method. The position of the symbols is consistent with the boundary lines by the MF method.

With any nonzero value of $U$, phase transition boundary lines can also be obtained analytically. For example, the boundaries between the solid $(0,1)$ to the TSF phase are:

$$
\mu=C \pm \frac{12 V+16 J}{3} \sqrt{\frac{3 U-20 J+3 V}{16 J+12 V}},
$$

where $C=U+2 V-16 J / 3$.

In Fig. 3 (c), we scan the phase diagram $U / V=3.5$ along $J / V=0.6$ and find that the above phase diagram is particle-hole symmetric by the transformation $\rho \rightarrow 3-\rho$. In the region $0<\rho<0.25$ and $2.75<\rho<3$, the system is in the TSF phase. In the range $0.25<\rho<2.75$, the density changes discontinuously, which means the phase transition between the TSF phase and the solid phases, and phase transitions between solid phases, are first order.

\section{B. ASS with $t / V \neq 0, W / V=0$ and $U / V=0$}

In Fig. 4(a), with a moderate atom hopping $t / V$, i. e., $t / V=0.5$, two phases namely the ASF and ASS phases emerge between the empty phase and the solid phase $(0,3)$. The phase transition from the ASF phase to the TSF phase is of first order due to the quantum fluctuations [15]. The phase transition from the ASS to the TSF phase is also first order.

To illustrate the results above in more detail, we scan the phase diagram along the lines $J / V=0.32,0.4$, and 0.65 , as shown in Figs. 4 (b)-(d), respectively. With $J / V=0.32$ and starting at $\mu / V=-2$, the system sits in a TSF phase with $\Psi^{t} \neq 0$ and $\Psi^{a}=0$. When increasing the chemical potential $\mu$, the system enters into the ASF phase discontinuously. The order parameter $\Psi^{a}$ jumps to a nonzero value, and $\Psi^{t}$ deceases steeply. The jump
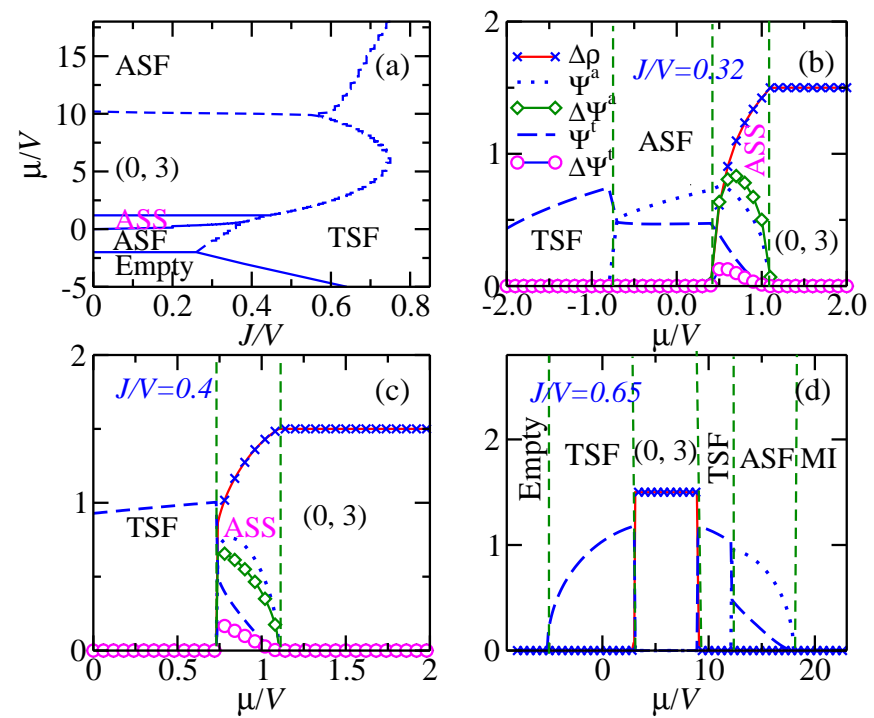

FIG. 4: (Color online) (a) MF phase diagram of the model represented by Eq. (7) for $U / V=0, t / V=0.5$, and $W / V=$ 0 . The dashed line and the solid line denote the first-order and the continuous phase transitions, respectively. Detailed description of $\Delta \rho, \rho, \Delta \Psi_{t}$, and $\Psi_{t}$ for the square lattice with $U=0, t=0$ and $W=0$ at (b) $J / V=0.32$ (c) $J / V=0.4$ and (d) $J / V=0.65$.

means the ASF-TSF phase transition is first order, which is consistent with our previous work [15]. In Fig. 4(c), the phase transition from the TSF to the ASS phase is first order while the phase transition from the ASF to the ASS phase is continuous as shown in Fig 4 (b). In Fig. 廿4(d), with $J / V=0.65$, the ASS phase disappears and the TSF and ASF phases dominate most of the phase diagram.

\section{TSF with $t / V=0, W / J=12$ and $U / V=0$}

In order to make the real ultra cold atom experiment easier to observe the TSF phase, we try to make the PA method drive the trimers active in the optical lattice. We don't try to combine the PA method with the magnetic Feshbach resonance method together to modulate the three-body interaction independently. The price of just using the PA method is that the ratio $W / J$ has to be kept at 12 and is not controllable.

Fortunately, we find in Fig. 5 (a) the TSF phase with $W / J=12$. In the larger $\mu$ region, the phase boundaries between the phases $(3,3),(0,3),(0,2)$ and $(3,2)$ are completely identical to the classical limit phase diagram in Fig. 2(b). In the lower $\mu$ region, the expected TSF phase appears only in the region $(\rho<2)$, in spite of the block from the competition from the nearest neighborhood interaction and the local three-body repulsive interaction. The numerical phase boundary between the $(0,2)$ solid to the TSF phase are very consistent with the analytical results: 

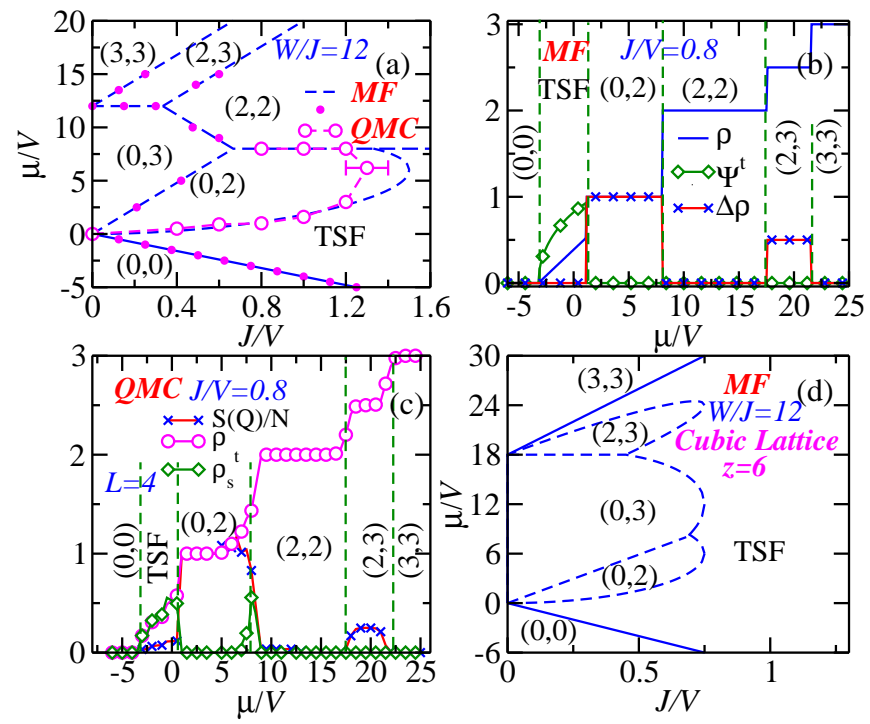

FIG. 5: (Color online) (a) MF and QMC phase diagram $(J / V, \mu / V)$ with $W / J=12$. (b) By the MF method, detailed description of the density $\rho$, the TSF order $\Psi^{t}$ and solid orders $\Delta \rho$ and $\Delta \Psi^{t}$ for the square lattice with $U=0, t=0$ and $W=$ $12 J$ at $J / V=0.8$. (c) By the QMC method, the density $\rho$, the structural factor $S(\mathbf{Q}) / N$, and the TSF density $\rho_{s}^{t}$ in the same parameters regimes as in (b). (d) The MF phase diagram for the cubic lattice with $z=6$ for comparison purposes.

$$
\mu=\frac{1}{6}\left((8 J+24 V) \pm \sqrt{(8 J+24 V)^{2}-576 J^{2}}\right) .
$$

At the same time, in the classical limit, the configuration $(0,3)$ disappears if $W / V>7.5$. However, in the quantum phase diagram, when $W / V>7.5$ the TSF phase exists. This implies that the TSF phase benefits from the relative strength of the trimer tunneling terms and the on-site repulsion is not the only interaction which determines the trimers in the TSF phase.

It should be pointed out that for the square lattice, theoretically, the TSF phase only exists in the range $W / J<24$ with just two nonzero parameters $W$ and $J$. Experimentally, it is possible to find the TSF phase as the ratio $W / J=12$ is smaller than 24 for arbitrary detuning parameter $\Delta$.

We describe the phase diagram in more detail along $J / V=0.8$ as shown in Fig. 5 (b). The system undergoes a phase transition from the empty phase to the TSF phase at $\mu / V=-3.1$. At $\mu / V=1.1$, the TSF phase disappears and the system enters into the $(0,2)$ solid phase with a first-order phase transition due to the jumps of the quantities $\Psi^{t}$ and $\rho$. When increasing the ratio $\mu / V$, the solid phases $(2,2),(2,3)$, and the MI phase $(3,3)$ appear successively. By the QMC method, Fig. 5(c) shows the details along $J / V=0.8$. The regions are very consistent with the results by the MF method as shown in Fig. 5. (b). In the region around $\mu / V=9, \rho_{s}^{t}$ is nonzero, but becomes zero in the thermaldynamical limit.
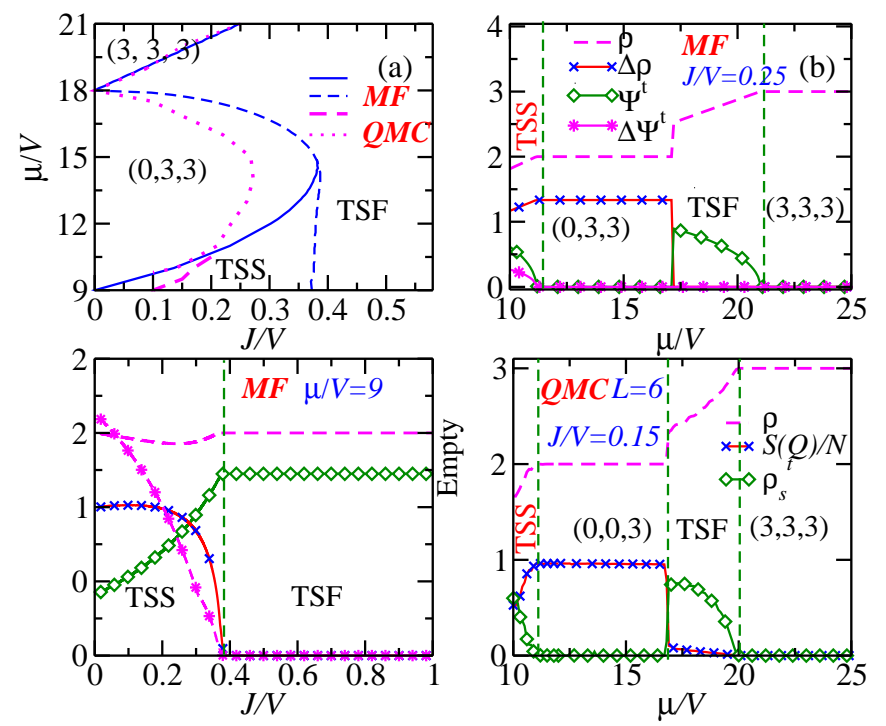

FIG. 6: (Color online) (a) MF and QMC phase diagram of the model represented by Eq. (6) for $U=0, t=0$ and $W=0$ for the triangular lattice. The dashed line and the solid line denote the first order and the continuous transitions, respectively. (b) By the MF method, detailed description of the density $\rho$, the TSF order $\Psi^{t}$, and the solid orders $\Delta \rho$ and $\Delta \Psi^{t}$, with $J / V=0.25$. (c) The same quantities as in (b) at $\mu / V=9$. (d) The density $\rho$, the structural factor $S(\mathbf{Q}) / N$, and the superfluid density $\rho_{s}^{t}$ with $J / V=0.15$ by the QMC method and the results are consistent with those in (b).

In Fig. 囵(d), for the purpose of comparison, we compute the quantum phase diagram on the cubic optical lattice with coordination number $z=6$. Without the next-nearest repulsion, the quantum phase diagram of the Bose-Hubbard on the lattices with different dimensions are similar. However, with off-site interactions, the phase diagrams need to be checked, as the coordination number $z=6$ is larger than the square lattice, it is easier for the kinetic-dominated TSF phase to emerge. Eventually, in contrast to the square lattice, we find the TSF phase in a wide range.

\section{TSS ON THE TRIANGULAR LATTICE}

\section{A. TSS with $U / V=0, t / V=0$ and $W / J=0$}

This section shows the phase diagram for $U=0$ on the triangular lattice. In Fig. 6(a), we show the phase diagram of the model described by Eq. (6) on the triangular lattice, which contains the empty, solid, TSF and MI phases. The new emerged phase is the TSS phase.

In the TSF phase, $\rho$ and $\Psi^{t}$ for every site are the same. By obtaining the lowest eigenvalue of Eq. (6), the trimer superfluid order parameter becomes:

$$
\Psi^{t}=\frac{\sqrt{(12 J+\mu)(12 J+18 V-\mu)}}{\sqrt{6}(4 J+3 V)} .
$$


The phase boundaries between the TSF phase and the solid (MI) phases can also be obtained analytically; these are not shown here.

We show the details of the phase diagram along the line $J / V=0.25$. By both the nonzero values of the solid orders $\Delta \rho$ and $\Delta \Psi^{t}$, and the TSF order $\Psi^{t}$, a stable TSS phase exists between the two solid phases, i.e.: in the range $10<\mu / V<11.4$. The mechanism is order by disorder 28], in which quantum fluctuations break the partially classical degeneracy. At the same time, the phase transition from the TSF phase to the solid phases are first order.

As shown in Fig. 6 (c), we scan the phase diagram along $\mu / V=9$. In the region $0<J / V<0.39$, the solid order and TSF order are robust and coexisting. The phase transition from the TSS to the TSF phases are continuous in the particle-hole symmetric point $\mu / V=9$ [46, 49]. As shown in Fig. 66 (d), we scan the phase diagram at $J / V=0.15$ by the QMC method. The result is again very consistent with Fig. 6 (b) by the MF method.

We also study the effects of the on-site interaction $U / V$, not shown. We start at the point $\mu / V=8$ and $J / V=0.25$ which is at the TSS phase. By increasing $U / V$, the TSS phase survives in a reasonable range and enters into the $(0,0,3)$ phase.

\section{B. TSS with $t / V=0, U / V=0$ and $W / J=12$}

Figures 7 shows the global phase diagram for the triangular lattice with $W / J=12$. When the ratio $J / V$ is relatively weak, several kinds of solid phases are found as shown in the classical limit phase diagram Fig. 2(d). As

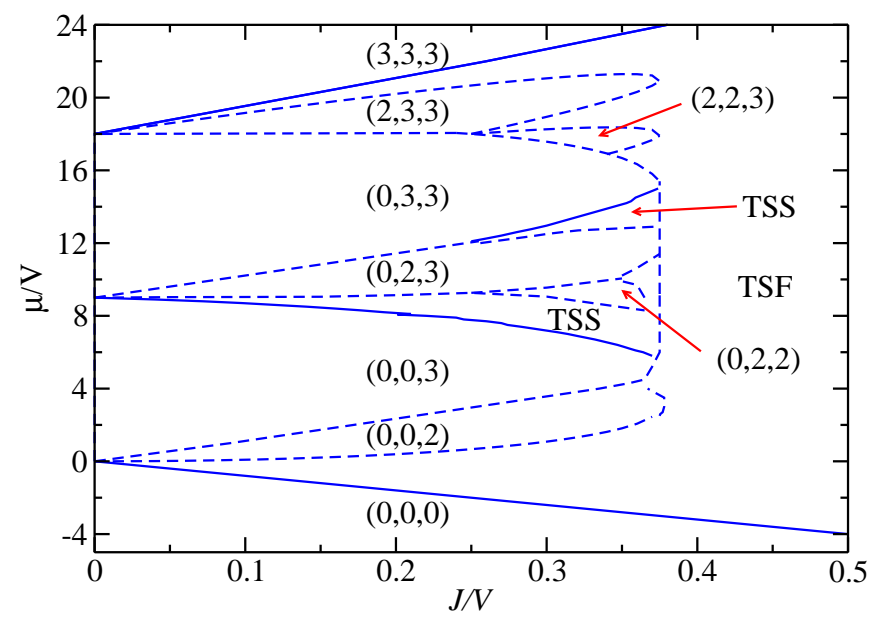

FIG. 7: (Color online) MF phase diagram of the triangular lattice for $U=0, t=0$ and $W / J=12$. The dashed line and the solid line denote the first order and the continuous transitions, respectively. The TSS phases exists in the two independent regions. In other words, two solid phases emerge in the original region of the TSS phase in Fig. 6(a).

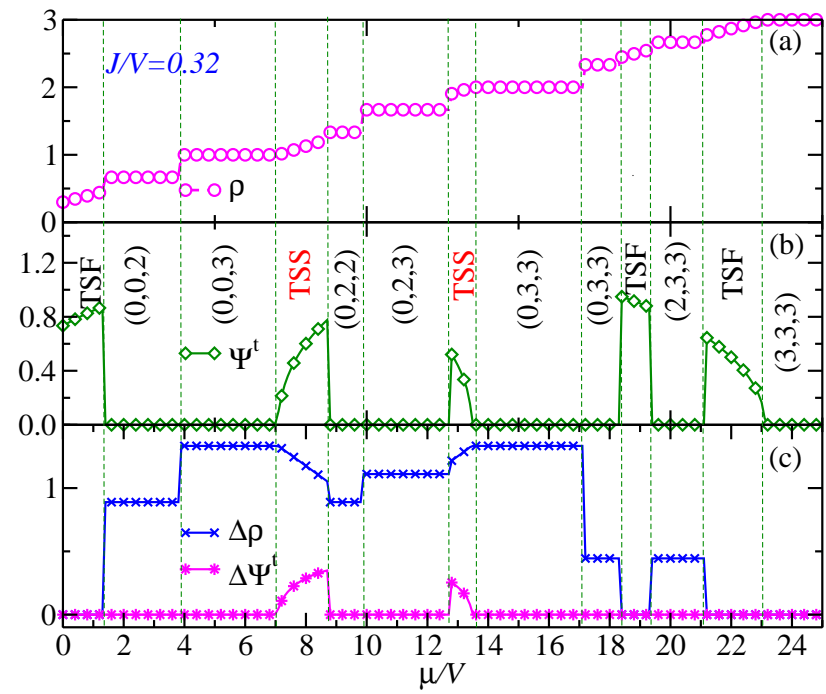

FIG. 8: (Color online) MF detailed description of the density $\rho$, the TSF order $\Psi^{t}$ and solid orders $\Delta \rho$ and $\Delta \Psi^{t}$, for the triangular lattice with $U=0, t=0, W / J=12$ and $J / V=$ 0.32. The TSS phases exist in the two regions $7.1<\mu / V<8.7$ and $12.8<\mu / V<13.4$.

the ratio $J / V$ become stronger, the expected TSF phase appears. In this phase diagram, the TSF phase can be found in the range $0<\rho<3$, which is significantly different from the case on the square lattice. All of the phase boundaries between the TSF phase and the solid phase obtained by the iteration method have been checked by analytical evaluation.

Fortunately, apart from the previous phases, we find two independent regions of the TSS phases. One may think this is an unexpected result because, in the classical limit phase diagram, the two types of solid $(0,0,3)$ and $(0,3,3)$ are not degenerate, which is the condition that the supersolid exists [28, 29, 43, 44]. However, the quasi trimer solid $(0,2,3)$ is degenerate with the trimer solid $(0,0,3)$.

Comparing with Fig. 6(a), the areas of the TSS phase become smaller and the solids $(0,2,3)$ and $(0,2,2)$ appear between the two regions of the TSS phases. Moreover, the phases $(0,0,2),(2,3,3)$ and $(2,2,3)$ emerge due to the break of the trimers by the three-body repulsion.

Figure 8 shows the detailed descriptions of the density $\rho$, the TSF order $\Psi^{t}$ and solid orders $\Delta \rho$ and $\Delta \Psi^{t}$ along $J / V=0.32$. The TSS phase exists in the range $7.1<\mu / V<8.7$ and $12.8<\mu / V<13.4$, for both $\Psi_{t}$ and $\Delta \rho$ are nonzero. Also there are several solid phases, in which $\Delta \rho$ and $\rho$ are in some horizontal platforms. Furthermore, two TSF phases appear in the three independent regions. Unlike the square lattice case, the density of the TSF phase covers the range $0<\rho<3$. The phase transitions from the TSF phase to the solid phases $(0,0,3)$ and $(0,3,3)$ are continuous, while the phase transitions from the TSF phase to the quasi trimer solid $(0,2,3)$ 
and the other phase $(0,2,2)$ are first order.

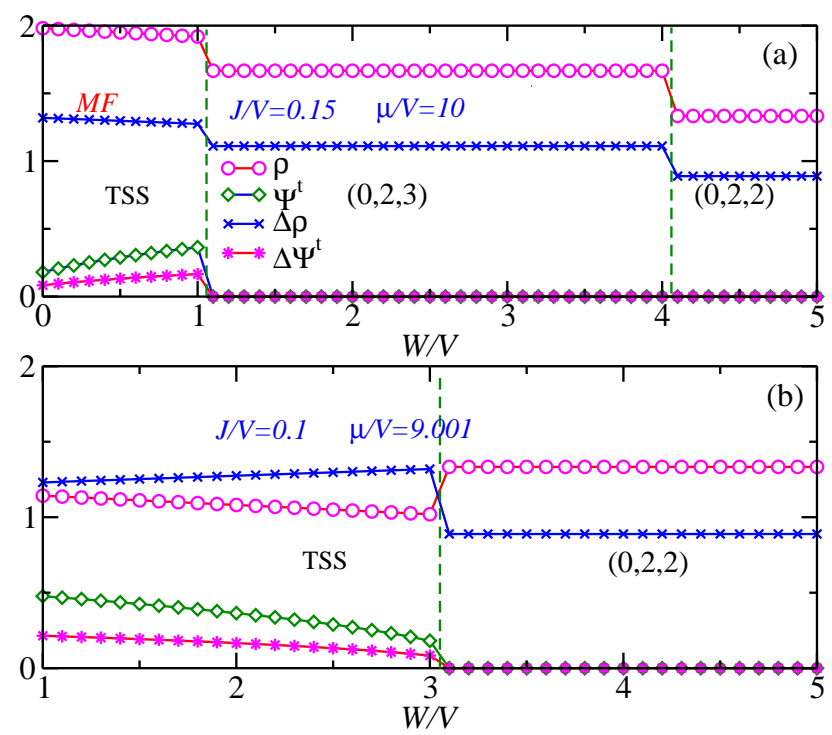

FIG. 9: (Color online) MF description of the density $\rho$, the TSF order $\Psi^{t}$, and solid orders $\Delta \rho$ and $\Delta \Psi^{t}$ as functions of $W / V$ at (a) $\mu / V=10$ and $J / V=0.15$, and (b) $\mu / V=9.001$ and $J / V=0.1$. The jumps of the order parameters confirm that the phase transition from TSS to the quasi trimer solid phase is first order.

To investigate the phase transition from the TSS phase to the quasi trimer solid phase, we also start a TSS phase, where has been proved in Fig. 6 (d), then increase the three-body interaction. Fig. 9 shows the quantities $\rho$, $\Delta \rho, \Psi^{t}$ and $\Delta \Psi^{t}$ as functions of $W$ at $\mu / V=10$ and $J / V=0.15$. From the jumps of these quantities around $W / V=1$, the phase transition from the TSS phase to the solid phase $(0,2,3)$ clearly is first order. We also find that the transition from the TSS to the solid $(0,2$, 2 ) is first order at $J / V=0.1$ and $\mu / V=9.001$ around $W / V=3.05$.

\section{DISCUSSION AND CONCLUSIONS}

Most of results in the present work were obtained by the MF method. This method ignores quantum fluctuation terms and correlation effects by a decoupling approximation [31, 32], but was nonetheless successful at predicting SS phases [45, 46] and phase transitions [47, 48], that were eventually confirmed by QMC calculations [49, 50] or the density matrix renormalization group method [51]. In this work, we checked our MF results by the QMC method in some applicable regions and found that the results from both methods were very consistent.

In conclusion, we obtain the phase diagram of the extended Bose-Hubbard model with explicit trimer tunneling terms and a three-body interaction, on both the square and the triangular lattices.

For the square lattice, with a fixed two-body interaction, a TSF exists if the trimer tunneling terms are large enough. With finite atom hopping, an ASS phase exists, and the phase transition between the ASS phase and TSF phase is of first order. With a nonzero three-body interaction $W / J=12$, the TSF phase exists with the $0<\rho<2$ if $J$ is not sufficiently large. For comparison, we calculated the cubic lattice with $z=6$, and the TSF phase emerges in the larger range $0<\rho<3$.

For the triangular lattice, without the three-body interaction, we find a stable TSF phase and also the TSS phase. In particular, with the three-body repulsive interaction, we also find a stable TSS phase, due to the classical degeneracy between the quasi trimer solid and the trimer solid being broken by the quantum fluctuation. The phase transitions from the TSS phase to the quasi trimer solid are first order and the phase transition from the TSS phase to the trimer solid becomes continuous. Our results will be helpful in realizing the TSF and TSS phases by cold atom experiments.

\section{Acknowledgments}

W. Zhang thanks Songsong Wang in checking some numerical results. W. Zhang is supported by the NSFC under Grant No.11305113, No.11204204, Foundation of Taiyuan University of Technology 120504020102. T. C. Scott is supported in China by the project GDW201400042 for the "high end foreign experts project". C. Ding is supported by the NSFC under Grant No.11205005, Foundation of Anhui Province $1408085 \mathrm{MA} 19$.
[1] N. Gross, Z. Shotan, S. Kokkelmans, and L. Khaykovich, Phys. Rev. Lett. 105, 103203 (2010); 103, 163202 (2009).

[2] S. E. Pollack, D. Dries, and R. G. Hulet, Science 326, 1683 (2009).

[3] M. Zaccanti et al., Nature Phys. 5, 586 (2009).

[4] R. J. Wild, P. Makotyn, J. M. Pino, E. A. Cornell, and D. S. Jin, Phys. Rev. Lett. 108, 145305 (2012).

[5] M. Berninger et al., Phys. Rev. Lett. 107, 120401 (2011); B. Huang, L. A. Sidorenkov, R. Grimm, and J. M. Hut- son, Phys. Rev. Lett. 112, 190401 (2014).

[6] S. Tung, K. Jimenez-Garcia, J. Johansen, C. V. Parker, and C. Chin, Phys. Rev. Lett. 113, 240402 (2014).

[7] K. Jones, E. Tiesinga, P. Lett, and P. Julienne, Rev. Mod. Phys. 78, 483 (2006); M. Stoll and T. Köhler, Phys. Rev. A 72, 022714 (2005).

[8] J. D. Miller, R. A. Cline, and D. J. Heinzen, Phys. Rev. Lett. 71, 2204 (1993).

[9] M. Mackie, M. Fenty, D. Savage, and J. Kesselman, Phys. 
Rev. Lett. 101, 040401 (2008); M. Junker, D. Dries, C. Welford, J. Hitchcock, Y. P. Chen, and R. G. Hulet, Phys. Rev. Lett. 101, 060406 (2008);L. H. Lu and Y. Q. Li, Phys. Rev. A 76, 053608 (2007).

[10] A. Zenesini, B. Huang, M. Berninger, H. Nägerl, F. Ferlaino, and R. Grimm, Phys. Rev. A 90, 022704 (2014).

[11] B. Huang, L. A. Sidorenkov, R. Grimm, and J. M. Hutson, Phys. Rev. Lett. 112, 190401 (2014).

[12] V. Efimov, Phy. Lett. B 33, 563 (1970).

[13] S. Piatecki and W. Krauth, Nat. Commun. 5, 3503 (2014).

[14] Y. Z. You, Z. Chen, X. Q. Sun, and H. Zhai, Phys. Rev. Lett. 109, 265302 (2012).

[15] W. Z. Zhang, R. Li, W. X. Zhang, C. B. Duan and T. C. Scott, Phys. Rev. A 90, 033622 (2014).

[16] S. Sachdev, Quantum Phase Transitions, (Cambridge University Press, 2003).

[17] O. Penrose and L. Onsager, Phys. Rev. 104, 576 (1956); A. F. Andreev and I. M. Lifshitz, Sov. Phys. JETP 29, 1107 (1969); G. V. Chester, Phys. Rev. A 2, 256 (1970); A. J. Leggett, Phys. Rev. Lett. 25, 1543 (1970).

[18] D. Rossini and R. Fazio, New J. Phys. 14, 065012 (2012).

[19] W. Z. Zhang, L. X. Li, and W. A. Guo, Phys. Rev. B 82, 134536 (2010).

[20] C. Trefzger, C. Menotti, and M. Lewenstein, Phys. Rev. Lett. 103, 035304 (2009).

[21] K. P. Schmidt, J. Dorier, A. Läuchli, and F. Mila, Phys. Rev. B 74, 174508 (2006); K. P. Schmidt, J. Dorier, A. M. Läuchli, and F. Mila, Phys. Rev. Lett. 100, 090401 (2008).

[22] H. C. Jiang, L. Fu, and C. K. Xu, Phys. Rev. B 86, 045129 (2012).

[23] W. Z. Zhang, R. X. Yin, and Y. C. Wang, Phys. Rev. B 88, 174515 (2013).

[24] C. M. Chung, S. A. Fang, and P. C. Chen, Phys. Rev. B 85, 214513 (2012).

[25] H. H. Hung, W. C. Lee and C. J. Wu, Phys. Rev. B 83, 144506 (2011).

[26] L. Pollet, J. D. Picon, H. P. Büchler, and M. Troyer, Phys. Rev. Lett. 104, 125302 (2010).

[27] G. Mazzarella, S. M. Giampaolo, and F. Illuminati, Phys. Rev. A 73, 013625 (2006).

[28] S. Wessel and M. Troyer, Phys. Rev. Lett. 95, 127205 (2005).

[29] M. Boninsegni and N. Prokof'ev, Phys. Rev. Lett. 95, 237204 (2005).
[30] Y. C. Wang, W. Z. Zhang, H. Shao, and W. A. Guo, Chin. Phys. B 22, 096702 (2013).

[31] D. van Oosten, P. van der Straten, and H. T. C. Stoof, Phys. Rev. A 63, 053601 (2001).

[32] X. C. Lu and Y. Yu, Phys. Rev. A 74, 063615 (2006).

[33] R. V. Pai, K. Sheshadri, and R. Pandit, Phys. Rev. B 77, 014503 (2008).

[34] A. Dhar, M. Singh, R. V. Pai, and B. P. Das, Phys. Rev. A 84, 033631 (2011).

[35] D. Lühmann, O. Jürgensen, M. Weinberg, J. Simonet, P. Soltan-Panahi, K. Sengstock, Phys. Rev. A 90, 013614 (2014).

[36] A. W. Sandvik, Phys. Rev. B 59, R14157 (1999). F. Alet, S. Wessel, and M. Troyer, Phys. Rev. E 71, 036706 (2005).

[37] O. F. Syljuåsen and A. W. Sandvik, Phys. Rev. E 66, 046701 (2002)

[38] L. Bonnes and S. Wessel, Phys. Rev. Lett. 106, 185302 (2011).

[39] L. Pollet, M. Troyer, K. Van Houcke, and S. M. A. Rombouts, Phys. Rev. Lett. 96, 190402 (2006).

[40] L. Bonnes and S. Wessel, Phys. Rev. B 85, 094513 (2012).

[41] K. K. Ng and M. F. Yang, Phys. Rev. B 83, 100511 (2011).

[42] E. L. Pollock and D. M. Ceperley, Phys. Rev. B 36, 8343 (1987).

[43] A. van Otterlo and K. H. Wagenblast, Phys. Rev. Lett. 72, 3598 (1994); A. van Otterlo et al., Phys. Rev. B 52, 16176 (1995); P. Sengupta, L. P. Pryadko, F. Alet, M. Troyer and G. Schmid, Phys. Rev. Lett. 94, 207202 (2005).

[44] M. Boninsegni and N. Prokof'ev, Rev. Mod. Phys. 84, 759 (2012).

[45] M. Iskin, Phys. Rev. A 83, 051606(R) (2011).

[46] D. Yamamoto, I. Danshita, and Carlos A. R. Sá de Melo Phys. Rev. A 85, 021601(R) (2012).

[47] T. Kimura, Phys. Rev. A 84, 063630 (2011).

[48] D. Yamamoto, G. Marmorini, and I. Danshita, Phys. Rev. Lett. 112, 127203 (2014).

[49] L. Bonnes and S. Wessel, Phys. Rev. B 84, 054510 (2011).

[50] T. Ohgoe, T. Suzuki, and N. Kawashima, Phys. Rev. B 86, 054520 (2012).

[51] D. Sellmann, X. Zhang, and S. Eggert, arXiv: 1403.0008 (2014). 